\title{
RBANS has reasonable test-retest reliability in schizophrenia
}

Wilk C, Gold J, Bartko J, Dickerson F, Fenton W, Knable M, Randolph C, Buchaanan R. Test-retest stability of the Repeatable Battery for the Assessment of Neuropsychological Status in schizophrenia. American Journal of Psychiatry 2002 May; 159 : 838-44.

QUESTION: What is the test-retest reliability of the Repeatable Battery for the Assessment of Neuropsychological Status (RBANS) in people with schizophrenia compared to healthy controls?

\author{
Design \\ Case-control study with repeated tests.
}

\section{Setting}

Three hospitals and psychiatric research centres, USA.

\section{Participants}

181 adults with schizophrenia or schizoaffective disorder and 99 healthy people. People with mental retardation, drug problems or alcohol dependence were excluded.

\section{Description of tests}

The RBANS is a cognitive screening test with a total score scale and five specific cognitive ability index scores. Participants completed one form of the RBANS followed by the alternate form at a later date. The interval between tests ranged from 1-134 days.

\section{Main outcome measures}

Test-retest reliability measured using intra-class correlation coefficients. The authors controlled for medication adjustments and other confounders.

\section{Sources of funding:}

Stanley Foundation.

For correspondence: Dr Gold, Maryland Psychiatric Research Center, Baltimore, USA. jgold@

mprc.umaryland.edu

\section{Main results}

The RBANS was sensitive to cognitive impairment in schizophrenia. People with schizophrenia had scores about 30 points lower than the comparison group. Testretest reliability correlations were 0.84 for people with schizophrenia and 0.77 for controls. Mean differences in retest scores were small across the two study groups

Test-retest reliability of RBANS scores for people with schizophrenia and healthy comparators

\begin{tabular}{|c|c|c|c|c|}
\hline & \multicolumn{2}{|c|}{ Association (correlation) } & \multicolumn{2}{|c|}{$\begin{array}{l}\text { Reliability (intraclass } \\
\text { correlation) }\end{array}$} \\
\hline & Schizoprenic & Comparison & Schizoprenic & Comparison \\
\hline Total scale & 0.84 & 0.78 & 0.84 & 0.77 \\
\hline Immediate memory & 0.69 & 0.55 & 0.67 & 0.55 \\
\hline $\begin{array}{l}\text { Visuospatial / } \\
\text { constructional } \\
\text { ability }\end{array}$ & 0.71 & 0.53 & 0.71 & 0.54 \\
\hline Language & 0.54 & 0.38 & 0.51 & 0.36 \\
\hline Attention & 0.81 & 0.77 & 0.81 & 0.76 \\
\hline Delayed memory & 0.69 & 0.57 & 0.68 & 0.53 \\
\hline
\end{tabular}

(none greater than 4 points). (Note from authors: The article provides base rate data on test-retest differences to guide clinical decision-making about cognitive change that can be applied to single case data).

\section{Conclusions}

Despite the brevity of the test, the Repeatable Battery for the Assessment of Neuropsychological Status (RBANS) has reasonable test-retest reliability in people with schizophrenia and healthy controls.

\section{COMMENTARY}

Neuropsychological assessment is becoming an essential part of clinical psychopathological examination, acting as a window into everyday mental processes in schizophrenia. ${ }^{1}$ Neuropsychological impairment affects functional outcome. It may be treated with novel antipsychotics and training programmes. Until recently, appropriate tests were sparse, especially short batteries that reduce attrition, but cover the most important areas of cognitive functioning. There have been several attempts to improve assessment, including the Repeatable Battery for the Assessment of Neuropsychological Status (RBANS). This paper is the third in a series assessing RBANS in schizophrenia. Previous publications found high sensitivity, reliability and validity. ${ }^{2-3}$

Although the study inconsistently 'crossed over' tests and included a heterogenous patient group, there are no major methodological problems. RBANS was originally developed for assessing dementia, ${ }^{4}$ therefore the battery does not necessarily have high specificity for cognitive impairment in schizophrenia. Instruments designed specifically for people with schizophrenia that include explicit testing of 'frontal functions' might have higher specificity and better sensitivity (for example the Brief Assessment of Cognition in Schizophrenia - BACS). Studies comparing RBANS with other short test batteries are required before introducing much needed new standards for people with schizophrenia. Bernhard Weber Dipl.-Psych., MD Department of Psychiatry and Psychotherapy Johann Wolfgang Goethe University Frankfurt, Germany

1 Keefe RS. The contribution of neuropsychology to psychia-

2 try. Am J Psychiatry 1995; 152: 6-15. the assessment of neuropsychological status as a screening the assessment of neuropsychological status as a screening
test in schizophrenia I: sensitivity, reliability, and validity. AmJ Psychiatry 1999; 156: 1944-50.
Pst in schizophrenia I: sensitivity,

3 Hobart MP, Goldberg R, Bartko JJ et al. Repeatable battery for the assessment of neuropsychological status as a screening test in schizophrenia, II: convergent/discriminant validity 156: 1951-7.

4 Randolph C. The Repeatable Battery for the Assessment of (RBANS): Preliminary Clinical Validity.J Clin Exp Neuropsychol 1998; 20: 310-9. 